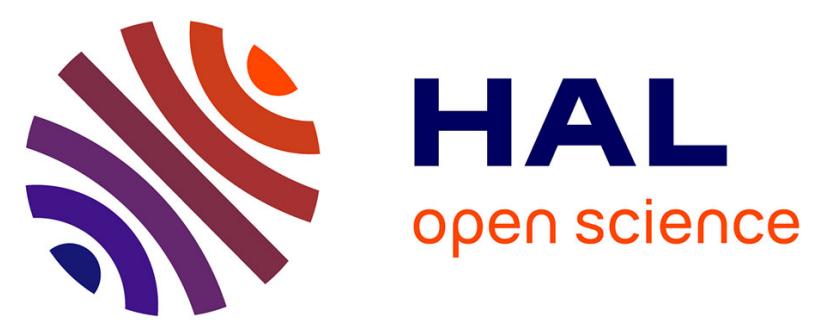

\title{
Dynamic fuel retention in tokamak wall materials: An in situ laboratory study of deuterium release from polycrystalline tungsten at room temperature
}

Régis Bisson, S Markelj, O Mourey, F Ghiorghiu, K Achkasov, J.-M Layet, P Roubin, G Cartry, C Grisolia, T Angot

\section{To cite this version:}

Régis Bisson, S Markelj, O Mourey, F Ghiorghiu, K Achkasov, et al.. Dynamic fuel retention in tokamak wall materials: An in situ laboratory study of deuterium release from polycrystalline tungsten at room temperature. Journal of Nuclear Materials, 2015, theme issue "Models and Data for Plasma-Material Interaction in Fusion Devices", 467 (1), pp.432. 10.1016/j.jnucmat.2015.07.028 . hal-01230492

\section{HAL Id: hal-01230492 \\ https://hal.science/hal-01230492}

Submitted on 26 Jan 2016

HAL is a multi-disciplinary open access archive for the deposit and dissemination of scientific research documents, whether they are published or not. The documents may come from teaching and research institutions in France or abroad, or from public or private research centers.
L'archive ouverte pluridisciplinaire $\mathbf{H A L}$, est destinée au dépôt et à la diffusion de documents scientifiques de niveau recherche, publiés ou non, émanant des établissements d'enseignement et de recherche français ou étrangers, des laboratoires publics ou privés.

\section{(ㅇ)(1) $\$$}

Distributed under a Creative Commons Attribution - NonCommercial - NoDerivatives $\mid 4.0$ 


\title{
Dynamic fuel retention in tokamak wall materials: an in situ laboratory study of deuterium release from polycrystalline tungsten at room temperature
}

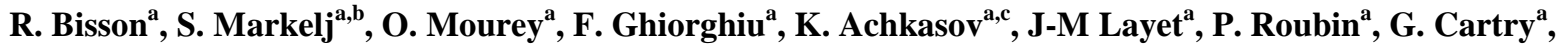 \\ C. Grisolia ${ }^{c}$ and T Angot ${ }^{\mathrm{a}}$ \\ ${ }^{a}$ Aix Marseille Université, CNRS, PIIM UMR 7345, 13397 Marseille, France \\ b Jožef Stefan Institute, Jamova cesta 39, 1000 Ljubljana, Slovenia \\ ${ }^{\mathrm{c}}$ CEA, IRFM, F-13108 Saint-Paul-lez-Durance, France
}

Corresponding author: regis.bisson@univ-amu.fr

\begin{abstract}
.
Retention of deuterium ion implanted in polycrystalline tungsten samples is studied in situ in an ultra-high vacuum apparatus equipped with a low-flux ion source and a high sensitivity thermodesorption setup. Retention as a function of ion fluence was measured in the $10^{17}-10^{21} \mathrm{D}^{+} \cdot \mathrm{m}^{-2}$ range. By combining this new fluence range with the literature in situ experimental data, we evidence the existence of a retention $\propto$ fluence $e^{0.645 \pm 0.025}$ relationship which describes deuterium retention behavior on polycrystalline tungsten on 8 orders of magnitude of fluence. Evolution of deuterium retention as a function of the sample storage time in vacuum at room temperature was followed. A loss of $50 \%$ of the retained deuterium is observed when the storage time is increased from 2 hours to 135 hours. The role of the surface and of natural bulk defects on the deuterium retention/release in polycrystalline tungsten is discussed in light of the behavior of the single desorption peak obtained with Temperature Programmed Desorption.
\end{abstract}

(C) 2015 EURATOM. Published by Elsevier B.V. This is an open access article under the CC-BY-NCND license.

This article appeared in the theme issue "Models and Data for Plasma-Material Interaction and Hydrogen Retention in Fusion Devices" 


\section{Introduction}

Fuel retention is one of the critical issues for plasma facing materials in fusion devices because of nuclear safety regulation related to the tritium inventory. This is the main reason why carbon plasma facing components (PFC) have been withdrawn in the final design of ITER, the international tokamak under construction in France, and tungsten has been chosen for constituting the divertor of ITER. Recently, the JET ITER-Like Wall (ILW) campaign [1] showed the benefit of tungsten and beryllium walls in terms of fuel retention as compared to the previously carbon-dominated configuration. However, the evolution of deuterium retention in the walls during operation and after discharges, i.e. the dynamic fuel retention, was different. Since in an ITER-like machine, two metals (beryllium and tungsten) with different retention behavior coexist, it is useful to perform laboratory experiments to understand separately the retention behavior of each metal. Furthermore, such laboratory experiments are helpful to develop wall models relevant for ITER-like fusion reactor.

In the literature, few laboratory experiments focused on deuterium dynamic retention in tungsten and all of them have been performed ex situ [2-4], i.e. the sample has been brought to air between ion implantation and dynamic retention quantification with thermo-desorption. As it has been shown recently by Wang et al. [5], air exposure complicates these retention measurements since numerous molecular species containing deuterium are generated and retention quantification can become inaccurate. This may explain why results between ex situ laboratory experiments on deuterium dynamic retention are somehow inconsistent. In situ studies of fuel retention are thus clearly needed. Very recently, Ohno et al. [6] have introduced an in situ and on-line deuterium retention measurement method using Nuclear Reaction Analysis (NRA). They were able to determine the evolution of deuterium concentration in graphite as plasma exposure was turned on and off. It was observed, respectively, an uptake and a loss of deuterium with a time constant on the minute range. However, this powerful method may be not the best one to study the dynamic retention of deuterium because there are some concerns regarding the intrusive aspect of Nuclear Reaction Analysis. Indeed, in this method the probe beam consists of ${ }^{3} \mathrm{He}$ ions with kinetic energy in the $\mathrm{MeV}$ range, being well above most materials displacement threshold energy and defects will be created $[7,8]$. This could generate a probe-induced evolution of the retention properties of sample materials which would complicate the interpretation of the obtained results.

In this article, a new in situ deuterium implantation/thermo-desorption setup is presented which allows absolute quantification of dynamic fuel retention in tungsten samples. By using low-flux low-energy deuterium ions impinging on recrystallized tungsten samples, a fully reversible system was obtained since absolute retention for a given set of experimental parameters remained within measurement uncertainties after more than a hundred experiments on the same sample. The confidence 
given by their reproducibility makes these results interesting for the development of Plasma-Material Interaction models. New data sets presented here, first, extend the retention versus fluence relationship 4 orders of magnitude towards low fluences, and second, demonstrate the existence of a $\sim 19$ hours time-scale for deuterium dynamic retention/release. While the former result could ease comparison with models on the microscopic scale, the latter result could prove important to assess fuel inventory after plasma discharges for tokamaks with ITER-like cooled divertors.

\section{Material and Experimental methods}

\subsection{Sample preparation}

Polycrystalline tungsten samples were provided by A.L.M.T. Corp. with a specified 99.99 wt.\% purity and cut into $10.0 \times 10.0 \times 0.4 \mathrm{~mm}^{3}$ specimens. Samples were delivered recrystallized with a typical grain size in the $\sim 30 \mu \mathrm{m}$ range and a mirror finish obtained by mechanical polishing. They were subsequently electro-polished in our laboratory with a $2.5 \mathrm{wt} . \% \mathrm{NaOH}$ solution and rinsed with distilled water. Samples were then mounted on a transfer platen made of molybdenum (purity $>99.9$ wt.\%, ultrasonically cleaned in acetone and ethanol). Once introduced in the Ultra High Vacuum (UHV) implantation/thermo-desorption chamber (base pressure $<4 \times 10^{-10}$ mbar), samples were subjected at least twice to a degassing procedure consisting of a linear temperature ramp of $1 \mathrm{~K} . \mathrm{s}^{-1}$ up to $1300 \mathrm{~K}$ followed by a 10 minutes annealing at $1300 \mathrm{~K}$.

\subsection{Ion implantation and sample storage}

Ion implantation of deuterium in the bulk of tungsten samples were performed with a Omicron ISE 10 Sputter Ion source fed with $\mathrm{D}_{2}$ gas (Air Liquide, N30 purity i.e. min. content of $99.9 \% \mathrm{D}_{2}$ ) through an all-metal leak valve. The majority deuterium ion species composing the ion beam was $\mathrm{D}_{2}{ }^{+}$ (>95\%), the minority species being $\mathrm{HD}^{+}, \mathrm{H}_{2}{ }^{+}$and $\mathrm{D}^{+}$, as verified with a quadrupole mass filter analyzer (Hiden EQP). Implanted ions impinged the sample with an angle of $45^{\circ}$ and with a kinetic energy of $500 \mathrm{eV}$, which corresponds to a kinetic energy per deuteron of about $250 \mathrm{eV}$. The ion beam flux was systematically determined by measuring the current going through the tungsten sample during ion implantation with a picoammeter (Keithley 410A). The size of the implanted area was defined by a collimating aperture resulting in a $0.5 \mathrm{~cm}^{2}$ beam spot at the sample position. The beam spot was measured using the ion-induced luminescence of a quartz sample which was recorded by a CMOS camera. The beam profile was that of a flat-top beam with an ellipsoidal elongation due to the 
$45^{\circ}$ incidence angle. The typical deuterium ion flux was $2 \times 10^{16} \mathrm{D}^{+} \cdot \mathrm{m}^{-2} \cdot \mathrm{s}^{-1}$ and therefore the sample temperature was not affected by the ion implantation. The deuterium ion fluence $\left(\mathrm{D}^{+} \cdot \mathrm{m}^{-2}\right)$ is obtained by multiplying the duration of the sample exposure to the monitored ion beam flux.

Since the ion source and the thermo-desorption oven are located in the same UHV chamber, the sample was transferred in a storage chamber (base pressure $<1 \times 10^{-9} \mathrm{mbar}$ ) after ion implantation. Then the oven was annealed until all dissociated $\mathrm{D}_{2}$ that eventually adsorbed on the oven was removed. Once the oven cooled down (typically >90 minutes), the tungsten sample was transferred back onto the oven and a thermo-desorption retention measurement could be realized.

\subsection{Temperature Programmed Desorption (TPD) and retention calibration}

Deuterium retention in tungsten sample is quantified using thermo-desorption. Our use of the technique is a Temperature Programmed Desorption (TPD) where the temperature of the implanted sample is linearly increased at a constant rate, while a Quadrupole Mass Spectrometer (QMS) located above the sample measures the desorption rate of molecules containing deuterium. Time integration of the QMS signal from deuterated molecular products allows quantifying the number of deuterium atoms which were present in the sample. Because TPD measurements are performed in situ, in the same apparatus than the ion implantation, contamination of the sample is drastically reduced and the majority of released species containing deuterium (typically $\geq 95 \%$ ) were $\mathrm{D}_{2}$ and $\mathrm{HD}$. The remaining deuterated product was DHO and it was found to be generated from the molybdenum platen. Therefore, $\mathrm{D}_{2}$ and $\mathrm{HD}$ are the only deuterated products accounted for in this work.

For TPD measurements, the sample was placed on top of the oven regulated with a PID controller (Eurotherm). Because our samples are transferable between multiple UHV chambers, we were not able to measure directly the sample temperature. Instead, we measured the temperature on the oven in the vicinity of the sample assembly with a K-type thermocouple. To obtain a linear temperature ramp on the tungsten sample, a non linear temperature ramp was programmed on the oven. The latter has been designed in the following way. In a first step, the PID controller was controlling a $1{\mathrm{~K} . \mathrm{s}^{-1}}$ ramp on a dummy sample thanks to a second K-type thermocouple attached to it. Both the dummy sample thermocouple and the oven thermocouple temperature measurements were recorded. In a second step, the PID controller was programmed with a non-linear function (a sequence of several linear ramps) to reproduce the oven thermocouple temperature excursion observed during the $1 \mathrm{~K}^{-1}{ }^{-1}$ dummy sample ramp. Running the regulation on the oven thermocouple with the designed non-linear program gave a $1 \mathrm{~K} . \mathrm{s}^{-1}$ temperature ramp on the dummy sample thermocouple with an 
excellent reproducibility. This non-linear program was used in this work to perform $1 \mathrm{~K} \cdot \mathrm{s}^{-1} \mathrm{TPD}$ ramp on tungsten samples.

In order to achieve high-sensitivity detection of desorbing species, a differentially pumped QMS (Hiden 3F/PIC) is used to collect directly the molecular species desorbing from the sample. The differential pumping chamber is home-designed and has a low background pressure $\left(<8 \times 10^{-11} \mathrm{mbar}\right)$. Conversion of the QMS signal into desorption rate from the sample necessitates to know the angular distribution of the released deuterium species, the geometric acceptance of the differential stage, and the QMS sensitivity for the considered species. The angular distribution of desorbing species is assumed to follow a cosine distribution. This hypothesis is based on the corrugated polycrystalline nature of the sample whose distributions of grain orientations (and cut misalignments with respect to crystalline planes) and azimuthal orientations should lead to a geometric smearing of the angular distribution of desorbing species. Such effects have been shown to significantly broaden angular distribution of scattered molecules [9-11] and molecules produced by recombination [12], especially when the surface contains defects and/or atomic steps [13]. The geometric acceptance of the differential pumping stage has been determined with Monte-Carlo particle trajectories simulation and depends on the ion beam footprint on the sample, the dimension of the aperture connecting the sample chamber and the QMS chamber, and the sample-aperture distance. The first has been measured (Section 2.2), the second is our design and has been checked with conductance measurements and the latter is measured thanks to the translation stage on which the oven is mounted. Finally, the QMS sensitivity is regularly calibrated by measuring the QMS signal as a function of a varying $\mathrm{D}_{2}$ pressure, set in the oven chamber by an off-axis leak valve, which can be converted into a flux of $\mathrm{D}_{2}$ entering the differential stage of the QMS thanks to the kinetic theory of gases. Since it is difficult to calibrate the QMS signal for HD products with the same method, we use instead the $\mathrm{D}_{2}$ calibration corrected from their relative ionization cross sections $[14,15]$ and the characterization of the mass dependent ion detection efficiency of our QMS [16].

TPD quantification of deuterium retention realized in our setup has been cross-checked with Nuclear Reaction Analysis (NRA) employing the $\mathrm{D}\left({ }^{3} \mathrm{He}, \mathrm{p}\right) \alpha$ nuclear reaction. Measurements were performed at the Jožef Stefan Institute (JSI) on well characterized and deuterium implanted samples prepared in Aix-Marseille Université (AMU). NRA deuterium depth profile up to $7 \mu \mathrm{m}$ was measured by employing five different ${ }^{3} \mathrm{He}$ energies from $0.77 \mathrm{MeV}$ to $4.3 \mathrm{MeV}$ [8]. The total deuterium concentration was obtained by integration of the deuterium concentration in the depth profile. Because NRA measurements are less sensitive than what offers the AMU TPD setup, an ion fluence of $1 \times 10^{21} \mathrm{D}^{+} . \mathrm{m}^{-2}$ was used. This high fluence corresponds to an ion implantation duration greater than 10 hours i.e. the dynamic retention/release discussed in the following (section 3.3. and 4) already 
significantly diminished the deuterium retention in the sample at the end of implantation. Therefore, the $\sim 30$ hours necessary to transfer the sample from AMU to JSI (in dry nitrogen atmosphere) should lead to less than a 50\% loss of the implanted deuterium for NRA measurements as compared to a TPD measurement performed 2 hours after implantation. On the other hand, NRA measurements is a more local measurement (the ${ }^{3} \mathrm{He}$ probe beam is $2 \mathrm{~mm}$ in diameter) than the TPD technique. However, for a given fluence/storage time combination, NRA and TPD should result in similar retention values since the ion implantation footprint is quite homogeneous (see section 2.2). All in all, the NRA and TPD retention measurements should agree within less than a factor of two, which is confirmed and will be presented in Section 3.2.

\section{Results}

\subsection{Deuterium retention with a single TPD desorption peak}

Deuterium ions with a kinetic energy of $250 \mathrm{eV} / \mathrm{D}^{+}$were implanted in polycrystalline tungsten samples at room temperature $(319 \pm 12 \mathrm{~K})$ with various fluences comprised between $6 \times 10^{17}$ and $2.6 \times 10^{21} \mathrm{D}^{+} . \mathrm{m}^{-2}$. Two hours after the end of ion implantation, the deuterium content of these samples was measured with TPD. In Figure 1, the typical evolution of the $\mathrm{D}_{2}$ desorption rate as a function of sample temperature during TPD is shown for a series of fluences. These TPD measurements present a single desorption peak, slightly asymmetric, with the high temperature tail being more pronounced than the low temperature one. The sample temperature at which the peak maximum occurs is constant for fluences above $1.4 \times 10^{19} \mathrm{D}^{+} \cdot \mathrm{m}^{-2}$. Below this fluence, the temperature position of the peak maximum shifts gradually by about $30 \mathrm{~K}$ to higher temperature as the fluence is decreased, being a significant shift since the reproducibility of the peak maximum for a given sample is within $10 \mathrm{~K}$.

The remarkable feature of these implantation and TPD experiments resides in the presence of a single desorption peak. This is at variance with the literature where, for similar implantation temperature and deuterium kinetic energy [17-19] two desorption peaks are generally observed, with the first maximum being around $450-500 \mathrm{~K}$. In our experiments, the single desorption peak has a desorption rate maximum located at $455 \pm 30 \mathrm{~K}$. The indicated uncertainty comes from sample to sample variability which has been linked to difference in tightening of the sample on the transfer platen. A second desorption peak was observed with our samples only when the electro-polishing preparation step was not well performed, which may explain the difference in the number of desorption peaks between our results and the ones from the literature.

Furthermore, when repeating the same implantation conditions, the shape and the area of this 
single desorption peak remained constant within the experimental uncertainties independently of the cumulated fluence on a given sample (up to $8 \times 10^{21} \mathrm{D}^{+} . \mathrm{m}^{-2}$ i.e. $>90$ implantation/retention sequences). This behavior indicates that the present deuterium implantation/TPD measurement sequences do not allow defects to build up in the sample and consequently the reversibility of our tungsten samples to their initial retention property is preserved. This makes our reproducibility stringent enough so that this data set can be used to constrain the development of deuterium retention models in tungsten.

\subsection{Deuterium retention as a function of ion fluence}

Figure 2 shows the absolute number of deuterium atoms retained in polycrystalline tungsten samples as a function of impinging deuterium ion fluence. Figure 2 summarizes only available in situ retention measurements, i.e. the new data set obtained at AMU $\left(10^{17}-10^{21} \mathrm{D}^{+} \cdot \mathrm{m}^{-2}\right.$ fluence, green solid circle, this work) and the one presented in 2003 by Ogorodnikova et al. from the Max-Planck-Institut für Plasmaphysik in Garching (IPP) $\left(10^{21}-10^{24} \mathrm{D}^{+} \cdot \mathrm{m}^{-2}\right.$ fluence, blue solid square, [17]). Note that AMU measurements systematically included a 2 hours storage time between deuterium implantation and TPD measurements whereas the storage time at the IPP [17] was 5 minutes. Another difference was the flux during implantation, being at least three orders of magnitude higher in [17] than at AMU.

As a guide for the eye, the grey dashed line indicates what would be the retention if all nonreflected ions would stay in the bulk of the material. To that purpose, the percentage of non-reflected ions has been determined to be $41 \%$ by using the Stopping and Range of Ions in Matter (SRIM) code [20] for our implantation conditions and assuming a pure W sample. Even though the measured retention at the lowest fluence does correspond to the SRIM calculation, deuterium retention for higher fluences does not follow a linear trend, consistently with previous laboratory experiments [17, 19]. Instead, deuterium retention follows roughly a square root proportionality with the deuterium fluence, or more precisely the following power law: retention $\propto$ fluence ${ }^{0.645 \pm 0.025}$, as shown in Figure 2 with the black dotted line. Note that the two highest fluences measured at AMU were not included in the fitting procedure as the corresponding implantation duration is $>10$ hours (see section 3.3). The determined power law relationship holds on 8 orders of magnitude and thus deuterium retention measured at AMU appears to be consistent with the retention behavior observed at IPP [17]. Nevertheless, in the common range of ion fluence $\left(10^{21} \mathrm{D}^{+} \cdot \mathrm{m}^{-2}\right)$ there is a quantitative discrepancy (by a factor of $2.7_{-0.8}^{+1.7}$ ) between AMU and IPP [17] measured deuterium retention, which is supported by NRA measurements performed at JSI on AMU samples (Fig. 2). Furthermore, it can be seen that our retention measurements follows the 0.64 power law until a fluence of $\sim 10^{21} \mathrm{D}^{+} . \mathrm{m}^{-2}$ after which measured retention departs from the power law and start to be lower. Such high fluences correspond to 
implantation durations longer than ten hours in our experimental apparatus. For example, in the common range of ion fluence of $10^{21} \mathrm{D}^{+} . \mathrm{m}^{-2}$, implantation durations at AMU are about a day while they are only of a few minutes at IPP [17], and this could be the cause of the apparent quantitative discrepancy between the two datasets. Thus, it is natural to wonder if deuterium retention in tungsten evolves over time scales of hours and days at the temperature used for implantation $(<330 \mathrm{~K})$, i.e. if retention is a dynamic process during long implantation.

\subsection{Dynamic retention of deuterium: retained fraction as a function of storage time}

In order to test the dynamic retention of deuterium in tungsten at room temperature, tungsten samples implanted with a fluence of $2.8 \times 10^{19} \mathrm{D}^{+} . \mathrm{m}^{-2}$ (corresponding to an ion bombardment duration of less than 30 minutes) were stored in UHV conditions at $300 \mathrm{~K}$ for various times before a TPD retention measurement was performed. Storage time varied from 2 hours to 135 hours. Results are presented in Figure 3. It can be seen that approximately half of the implanted fraction of impinging deuterium is lost when the storage time is greater than 40 hours, which is an evidence for the release / dynamic retention of deuterium in polycrystalline tungsten at room temperature. Dynamic retention has been consistently observed for several samples as well as for other fluences $\left(5.8 \times 10^{19} \mathrm{D}^{+} \cdot \mathrm{m}^{-2}\right.$ and $12.1 \times 10^{19} \mathrm{D}^{+} . \mathrm{m}^{-2}$, not shown).

Furthermore, the loss of implanted deuterium from tungsten occurs in a non-linear fashion. First, it drops rapidly in the first 20 hours and then slowly decreases until a nearly constant fraction of deuterium remains in the sample after 60 hours. This deuterium retention evolution is well adjusted with the relationship retention $\propto$ storage time $e^{-0.23}$ or an exponential decay of the following form:

$$
\text { retention }(t)=B+S \times \exp \left(-\frac{t}{\tau}\right)
$$

where $(B+S)$ is the retention just after implantation, $t$ is the storage time and $\tau=19 \pm 5$ hours. The physical interpretation of this expression will be developed in the discussion section.

\section{Discussion}

An original result of this study is that the TPD behavior of polycrystalline tungsten samples remained unchanged even after a hundred of retention measurements on the same sample. This reversibility indicates that the implantation/thermo-desorption sequences do not build up additional defects in the bulk of the material nor modify the role of the surface layer. It has been noticed that for all studied samples, the mirror-like finish of the samples surface started to be tarnished after several tens of implantation/thermo-desorption sequences. Nevertheless, the modification of the macroscopic 
surface reflectivity did not change the retention properties neither the TPD peak shape of implanted samples. This behavior suggests that the sample surface might not be associated to the rate limiting step of the deuterium release process. This proposition will be discussed in the remaining of the discussion as well as its implications.

Firstly, the observation of a shift of the TPD peak maximum by $30 \mathrm{~K}$ to lower temperature as the fluence (the retention) is increased (Section 3.1, Figure 1) could be consistent with a second order desorption kinetic where surface recombination of deuterium atoms is the rate limiting step. In that case, the $\mathrm{D}_{2}$ desorption rate obeys the following Polanyi-Wigner equation:

$$
r=-\frac{d \theta}{d T}=A \times \theta^{n} \times e^{-\frac{E_{a}}{k_{B} T}}
$$

where $r$ is the $\mathrm{D}_{2}$ desorption rate, $A$ is the attempt frequency divided by the linear heating rate, $\theta$ is the deuterium surface coverage, $n=2$ is the kinetic order, $E_{a}$ is the activation energy for the release process, $k_{B}$ is the Boltzman constant and $T$ is the surface temperature. Indeed, according to Redhead's analysis of this expression [21], the temperature position of the TPD peak maximum decreases with increasing the surface coverage $\theta$ and the lowest temperature position is attained when the deuterium surface saturation coverage is reached. As it is shown in Figure 1, the TPD peak position stops to shift to lower temperature for ion fluence equal or greater than $2.8 \times 10^{19} \mathrm{D}^{+} \cdot \mathrm{m}^{-2}$. This fluence would set the lowest fluence for which surface saturation is reached during a TPD measurement because the bulk deuterium content should act as a reservoir that keeps the surface coverage at saturation as the fluence is further increased. Thus for higher fluences than $2.8 \times 10^{19} \mathrm{D}^{+} \cdot \mathrm{m}^{-2}$, the term $\theta^{n}$ would be constant in Equation 2 and the desorption rate $r$ would become only proportional to the exponential part of the expression. This would make the desorption rate increased once the saturation coverage is reached and the TPD peak maximum would shift to higher temperature for implanted deuterium fluence higher than $2.8 \times 10^{19} \mathrm{D}^{+} . \mathrm{m}^{-2}$. This TPD peak behavior is not observed in our experiments, therefore we could be in position to discard a second order desorption kinetic from a rate-limiting surface process. However, we should first exclude other physical process to be responsible for the observed behavior since the deuterium/tungsten system studied here is not solely a gas-surface system but a gas-surfacebulk system, i.e. bulk diffusion is a process that should be accounted for. Since our experiments are performed at a constant flux, to increase the fluence we incremented the duration of implantation. Thus as the fluence is increased, the implanted species have more time to diffuse in the bulk of the material during implantation. For the same type of trap, the TPD peak position for deep-in-the-bulk deuterium will be higher in temperature than that of near-to-the-surface deuterium. Therefore, it is expected that diffusion would shift the TPD peak to higher temperature for higher fluence (long implantation duration). When we increased the fluence from $2.8 \times 10^{19} \mathrm{D}^{+} \cdot \mathrm{m}^{-2}$ to $3.2 \times 10^{20} \mathrm{D}^{+} \cdot \mathrm{m}^{-2}$, 
increasing the implantation duration for about 4 hours, the TPD peak did not shift anymore. Comparison of TPD peak positions obtained for a fluence of $2.8 \times 10^{19} \mathrm{D}^{+} \cdot \mathrm{m}^{-2}$ after 2 hours or 5 hours storage time (not shown) indicates that 3 hours of diffusion at room temperature is only able to induce a TPD peak shift of at most $10 \mathrm{~K}$ ( i.e. within our uncertainty on relative peak positions). Therefore, the magnitude of the upward peak shift induced by diffusion $(\leq 10 \mathrm{~K})$ above a fluence of $2.8 \times 10^{19}$ $\mathrm{D}^{+} \cdot \mathrm{m}^{-2}$ is much less important that the magnitude of the downward peak shift induced by increasing the fluence $(30 \mathrm{~K})$ for a fluence below $2.8 \times 10^{19} \mathrm{D}^{+} . \mathrm{m}^{-2}$. Thus, it is very unlikely that bulk diffusion would compensate for further lowering of the TPD peak maximum as the fluence is increased above $2.8 \times 10^{19} \mathrm{D}^{+} \cdot \mathrm{m}^{-2}$.

To summarize, since an upward shift of the TPD peak does not occur after the TPD peak stabilization at $455 \mathrm{~K}$ and because diffusion should not significantly interfere with a further shift to lower temperature of the TPD peak with increasing fluence, a rate-limiting mechanism with a second order desorption kinetic is not able to render our experimental observations. Therefore, these results strongly suggest that surface recombination is not the rate-limiting step in deuterium release from the bulk of polycrystalline tungsten.

Secondly, since the surface layer appears not to be associated with the rate limiting step for deuterium release in these samples, we turn to the bulk properties of the polycrystalline tungsten. Because of the retention reversibility of our samples, one can deduce that the measured TPD peak should be related to natural bulk defects such as single vacancies, grain boundaries or dislocations. To definitely attribute the TPD peak to one of these bulk defects (and exclude the others) one would need a complete set of comparative measurements, using single crystals for probing the role of grain boundaries and high temperature annealing for varying dislocations concentration. This is an extended experimental program in itself and it is not the subject of the present study. However our results can be discussed in view of the recent theoretical investigations at the $a b$ initio level for the interaction of deuterium with natural bulk defects in tungsten such as single vacancy [22-27], grain boundary [28,29], and dislocation [29]. All these defects can accommodate several deuterium atoms in their vicinity. At $300 \mathrm{~K}$, it is expected $[23,26,27,29]$ that up to $5 / 6,3$ or 4 deuterium atoms can be trapped per single vacancy, grain boundary or dislocation sites, respectively. Furthermore, for these three types of bulk defects, the more deuterium atoms are bound to the defect, the less binding energy there is per deuterium atom. Therefore, in a TPD experiment these theoretical predictions would be evidenced in the following manner: as one would repeat an implantation/TPD experiment with increasing deuterium density (fluence) in the tungsten sample, the binding energy per deuterium would decrease and thus the temperature position of the TPD peak maximum would decrease too. In Figure 1, it is seen that the temperature of the TPD peak maximum decreases with increasing the fluence which follows 
qualitatively the trend inferred here from theoretical results. For a more quantitative analysis of the relation between TPD peak position (binding energy) and TPD peak area (retention is related to density), one would need to compare the density of natural defects with the density of deuterium atoms susceptible to decorate these defects. In the present experimental conditions, the maximal deuterium density in the implantation range (i.e. neglecting diffusion) should be in the $10^{-3}-10^{-4}$ range for the lowest fluence probed here. This puts an upper limit to the natural defect density that should be responsible for the TPD peak shifting behavior with increasing fluence evidenced in the present experiments. We hope this estimation could help the development of further theoretical studies aiming at identifying natural defects responsible for deuterium retention in polycrystalline tungsten.

Thirdly, we will attempt to identify which of the three proposed bulk defect candidates could exhibit a behavior consistent with our experimental results. We recall that the dynamic retention presented in Figure 3 has a time evolution which is well described by the exponential decay of Equation 1. This equation can be physically interpreted as the manifestation of the diffusion of implanted deuterium atoms in tungsten. Such diffusion is, in first approximation, isotropic and thus one can split the deuterium content in the sample after implantation in two populations; the deuterium diffusing deeper in the bulk and staying in tungsten (the $B$ parameter in Eq. 1, for "Bulk") and the deuterium diffusing towards the surface (the $S$ parameter, for "Surface") which will be released from the material following its recombinative desorption. Using $B=S$ allows fitting the data in Figure 3, which might seem consistent with a one dimensional isotropic diffusion, but this equality should not necessarily hold if anisotropic diffusion arises from the solubility limit of deuterium in tungsten. However, this problematic is out of the scope of this study and in the following we shall focus only on the desorbing deuterium population which is described in Equation 1 by the second term : $\left(S \cdot \exp \left(-\frac{t}{\tau}\right)\right)$. This functional form corresponds to the one obtained when performing an isothermal desorption of a system rate-limited by a first order kinetic process (by integration of Equation 2 with $n=1$ ). The decay of the deuterium retention in tungsten presented in Figure 3 has been obtained with the sample stored at a constant temperature $T_{\text {storage }}=300 \mathrm{~K}$, i.e. it was subjected to an isothermal desorption. It is expected that deuterium atoms de-trap from a bulk defect one by one and both [27] their activation energy $E_{a}$ and attempt frequency $A$ are sensitive to the number of deuterium around the trap i.e. the "trap coverage" $\theta$. It seems therefore reasonable to consider deuterium release from a bulk defect as a first order kinetic mechanism. Analyzing the observed exponential decay of the trapped population of Figure 3 as an isothermal desorption of a first-order kinetic process, we can estimate the mean activation energy for escaping the natural defect with the following equation: 


$$
\text { for } n=1, E_{a}=k_{B} \times T_{\text {storage }} \times \ln (A . \tau)
$$

Using the average attempt frequency calculated by Fernandez et al. [27], $A=1 \times 10^{13} s^{-1}$, and the experimental exponential decay of $\tau=19 \pm 5$ hours (Figure 3), we estimate that the deuterium release rate limiting step has a mean activation energy of $E_{a}=1.06 \pm 0.01 \mathrm{eV}$. We note that this value is consistent with the recent report by Watanabe et al. [30] of a deuterium release on a sub-hour time scale as measured with in situ NRA. A decay time constant of $\tau \sim 40$ minutes was found at $T_{\text {storage }} \cong 350 \mathrm{~K}$ in [30]. If we analyze this result along our proposition of a first order kinetic isothermal desorption, we extract an activation energy of $E_{a}=1.14 \mathrm{eV}$. The slightly higher activation energy could be related to the presence of additional defects induced by the $\mathrm{MeV}{ }^{3} \mathrm{He}$ beam used in NRA in [30] or could be the results of the time resolution of this in situ NRA setup (10 min).

These experimentally derived activation energies are consistent with those calculated by $a b$ initio methods $\left(E_{a}^{\sim} \sim 1.1 \mathrm{eV}\right)$ for a single vacancy containing 5 deuterium atoms $[23,26,27]$ or a $\sum 3(111)$ tilt grain boundary filled with 3 deuterium atoms [29], i.e. that are saturated at room temperature. Uncertainties on DFT activation energy values are usually estimated to be on the $10 \%$ level thus our experimentally estimated detrapping activation energy could be also consistent with the detrapping from a single vacancy containing 4 or 3 deuterium atoms $\left(E_{a} \sim 1.2 \mathrm{eV}\right)$ or a $(100)$ dislocation loop $\left(E_{a} \sim 1.0 \mathrm{eV}\right)$ filled with 3 deuterium atoms [29]. However for the (100) dislocation loop, de-trapping energies for the two last bound deuterium atoms are $E_{a} \geq 1.9 \mathrm{eV}$ which is inconsistent with the maximum temperature at which we observe deuterium release at low fluence in Figure 1 ( $600 \mathrm{~K})$. On the contrary, the last bound deuterium atoms in single vacancy and $\sum 3(111)$ tilt grain boundary are in the $E_{a} \sim 1.4-1.6 \mathrm{eV}$ range which is consistent [27] with our TPD observation at low fluence. Thus single vacancy and grain boundary are the two tungsten natural defects that can rationalize the observed dynamic retention i.e. the release of deuterium observed at room temperature. The single vacancy defect could also quantitatively explain our observation of a release of half of the retained deuterium on a time scale of a hundred hours since 5D-, 4D- (and 3Dfor some authors) -vacancy complexes have activation energy within $10 \%$ of $1.1 \mathrm{eV}$. Therefore, starting with a sample implanted with 5 deuterium per vacancy, one would obtain 3 ( or 2) deuterium per vacancy after an isothermal desorption of a hundred hour at room temperature. The last half of implanted deuterium atoms would stay trapped because their binding energy is $E_{a} \geq 1.4$ i.e. thermally stable at $300 \mathrm{~K}$.

Finally, we turn back to the reason that led us to evidence experimentally this dynamic retention. Initially, we aimed to rationalize quantitatively the discrepancy of Figure 2 between the 
AMU and the IPP data in the $10^{21} \mathrm{D}^{+} \cdot \mathrm{m}^{-2}$ fluence range. Considering the observed retention decay time constant of $\tau=19 \pm 5$ hours, we are able to account for a discrepancy by a factor of only $1.4 \pm 0.1$ i.e. lower than the observed $2.7_{-0.8}^{+1.7}$ factor. Our interpretation for this difference is that, as the fluence is increased, there is a more important loss of trapped deuterium after implantation that what is measured in our present setup. Indeed, in the recent work of You et al. [26] and Fernandez et al. [27], it was calculated that at room temperature up to 6 deuterium atoms could be bound to a single vacancy. This configuration has a calculated detrapping energy of $E_{a} \sim 0.9 \mathrm{eV}$ which would lead to a retention decay time constant on the minute time scale at $300 \mathrm{~K}$. Such a short time constant for dynamic retention will affect differently the AMU and the IPP setups since the data at AMU were systematically measured 2 hours after implantation while at the IPP the storage time was only 5 minutes. The overall effect would be that for the same high fluence needed to put 6 deuterium atoms in a single vacancy, the AMU measured retention will be $\frac{5}{6}$ smaller than at IPP, because a 2 hours storage time will be sufficient to release the $6^{\text {th }}$ deuterium atoms from single vacancy before the TPD retention measurement is performed. This will allow accounting for a discrepancy of $1.7 \pm 0.1$ which almost falls within uncertainties limit of the observed $2.7_{-0.8}^{+1.7}$ factor.

To conclude, both the TPD peak behavior with increasing fluence and the measured dynamic retention can be rationalized from the energetic of deuterium trapping on single vacancy and/or grain boundaries calculated by DFT ab-initio methods. Analysis of some of these theoretical works suggests that a further release of deuterium can be expected on a minute time scale at room temperature since up to 6 deuterium atoms can be trapped in tungsten single vacancy bulk defects at $300 \mathrm{~K}[26,27]$. A modification of our experimental setup is planned in order to address precisely this point. We believe that our experimental results demonstrate the importance of deuterium trapping on natural defects of tungsten and we expect that this would help to rationalize the physical mechanism behind the retention $\propto$ fluence $e^{0.645}$ law at $300 \mathrm{~K}[19,31,32]$ and would allow safer extrapolation of the tritium wall inventory for ITER.

As a closing remark, one could wonder how we managed to obtain a single desorption peak in the present set of experiments, in contrast to the previous literature. We underlined that we paid special attention to the electropolishing and to sample/beam purities. But one should also remember that, first, we used a low-flux ion beam, and second, we avoided thermal gradient in the vicinity of the ion beam footprint. While currently it is not possible to say if one of these specific parameters was most essential to our results, it should stimulate future studies dedicated to understand possible origins of the high desorption temperatures of implanted deuterium in tungsten materials. 


\section{Summary}

Deuterium retention in polycrystalline tungsten has been studied in a new in situ thermodesorption apparatus with low ion flux implantation capability. A reversible implantation/desorption sequence with a single desorption peak of deuterium molecular species has been obtained which allowed us to study the deuterium retention dependency on ion fluence and storage time with unprecedented sensitivity. When combining the presently measured fluence dependency with in situ data from Ogorodnikova et al. [17], we are able to evidence a retention $\propto$ fluence $e^{0.645 \pm 0.025}$ relationship that holds on the $10^{17}-10^{24} \mathrm{D}^{+} \cdot \mathrm{m}^{-2}$ fluence range. Furthermore, the storage time dependency measured at room temperature reveals that dynamic retention of deuterium in tungsten occurs on time scales of hours and days. Considering this deuterium release at $300 \mathrm{~K}$ together with the evolution of the temperature of the maximum desorption rate in TPD measurements, the origin of the retention in recrystallized polycrystalline sample has been discussed in terms of natural defects such as single vacancies or grain boundaries which can bind several deuterium atoms per defect site. This interpretation, based on recent DFT calculations, suggests that dynamic retention on shorter time scales (minutes) could occur. This broad range of deuterium release time scales (minutes to days) should be of importance for present and future tokamaks with actively cooled tungsten divertors, as both the control of the plasma and the working of tritium recycling plant could be affected. Further laboratory experiments are needed to definitely attribute the origin of this retention as well as its dynamics.

\section{Acknowledgments}

The authors acknowledge the work of Othmen Saidi as well as useful discussions with Yves Ferro. Financial support was provided by the French National Research Agency (ANR), through the project CAMITER (ANR-06-BLAN-0008), and the EURATOM-MESCS association (Slovenia). This work has been carried out within the framework of the EUROfusion Consortium and has received funding from the European Union's Horizon 2020 research and innovation programme under grant agreement number 633053. The views and opinions expressed herein do not necessarily reflect those of the European Commission. Part of this work has been carried out thanks to the support of the A*MIDEX project ( $n^{\circ}$ ANR-11-IDEX-0001-02) funded by the "Investissements d'Avenir" French Government program, managed by the French National Research agency (ANR). 


\section{References}

[1] S. Brezinsek, T. Loarer, V. Philipps, H.G. Esser, S. Grünhagen, R. Smith, R. Felton, J. Banks, P. Belo, A. Boboc, J. Bucalossi, M. Clever, J.W. Coenen, I. Coffey, S. Devaux, D. Douai, M. Freisinger, D. Frigione, M. Groth, A. Huber, J. Hobirk, S. Jachmich, S. Knipe, K. Krieger, U. Kruezi, S. Marsen, G.F. Matthews, A.G. Meigs, F. Nave, I. Nunes, R. Neu, J. Roth, M.F. Stamp, S. Vartanian, U. Samm, J.E. Contributors, Nucl. Fusion 53 (2013) 083023. [2] R. Sakamoto, T. Muroga, N. Yoshida, Journal of Nuclear Materials 233-237, Part 1 (1996) 776.

[3] A.D. Quastel, J.W. Davis, A.A. Haasz, R.G. Macaulay-Newcombe, Journal of Nuclear Materials 359 (2006) 8.

[4] K.A. Moshkunov, K. Schmid, M. Mayer, V.A. Kurnaev, Y.M. Gasparyan, Journal of Nuclear Materials 404 (2010) 174.

[5] P. Wang, W. Jacob, L. Gao, T. Dürbeck, T. Schwarz-Selinger, Nuclear Instruments and Methods in Physics Research Section B: Beam Interactions with Materials and Atoms 300 (2013) 54.

[6] N. Ohno, Y. Nakamura, M. Yamagiwa, T. Kaneko, N. Matsunami, S. Kajita, M. Takagi, Physica Scripta T159 (2014) 014053.

[7] G.M. Wright, D.G. Whyte, B. Lipschultz, Journal of Nuclear Materials 390-391 (2009) 544.

[8] S. Markelj, O.V. Ogorodnikova, P. Pelicon, T.S. Selinger, P. Vavpetič, I. Čadež, Phys. Scr. 2014 (2014) 014047.

[9] J.C. Tully, J. Chem. Phys. 92 (1990) 680.

[10] S. Ueno, Y. Narita, A.R. Khan, Y. Kihara, A. Namiki, Surface Science 602 (2008) 1585.

[11] R. Bisson, L. Philippe, M. Châtelet, Surface Science 600 (2006) 4454.

[12] R. Bisson, L. Philippe, M. Chatelet, P. Kratzer, J. Chem. Phys. 128 (2008) 017101.

[13] K.D. Rendulic, A. Winkler, H.P. Steinruck, Surface Science 185 (1987) 469.

[14] D. Rapp, P. Englander-Golden, The Journal of Chemical Physics 43 (1965) 1464.

[15] J.-S. Yoon, Y.-W. Kim, D.-C. Kwon, M.-Y. Song, W.-S. Chang, C.-G. Kim, V.

Kumar, B. Lee, Rep. Prog. Phys. 73 (2010) 116401.

[16] K. Achkasov, R. Bisson, T. Angot, P. Roubin, J.-M. Layet, A. Gicquel, J. Achard, A. Simonin, G. Cartry, to be published.

[17] O.V. Ogorodnikova, J. Roth, M. Mayer, Journal of Nuclear Materials 313-316 (2003) 469.

[18] O.V. Ogorodnikova, T. Schwarz-Selinger, K. Sugiyama, T. Dürbeck, W. Jacob, Phys. Scr. 2009 (2009) 014053.

[19] Z. Tian, J.W. Davis, A.A. Haasz, Journal of Nuclear Materials 399 (2010) 101.

[20] J.F. Ziegler, M.D. Ziegler, J.P. Biersack, Nuclear Instruments and Methods in Physics Research Section B: Beam Interactions with Materials and Atoms 268 (2010) 1818.

[21] P.A. Redhead, Vacuum 12 (1962) 203.

[22] Y.-L. Liu, Y. Zhang, H.-B. Zhou, G.-H. Lu, F. Liu, G.-N. Luo, Phys. Rev. B 79 (2009) 172103.

[23] K. Heinola, T. Ahlgren, K. Nordlund, J. Keinonen, Phys. Rev. B 82 (2010) 094102.

[24] D.F. Johnson, E.A. Carter, Journal of Materials Research 25 (2010) 315.

[25] K. Ohsawa, J. Goto, M. Yamakami, M. Yamaguchi, M. Yagi, Phys. Rev. B 82 (2010) 184117.

[26] Y.-W. You, X.-S. Kong, X.-B. Wu, Y.-C. Xu, Q.F. Fang, J.L. Chen, G.-N. Luo, C.S. 
Liu, B.C. Pan, Z. Wang, AIP Advances 3 (2013) 012118.

[27] N. Fernandez, Y. Ferro, D. Kato, Acta Materialia 94 (2015) 307.

[28] H.-B. Zhou, Y.-L. Liu, S. Jin, Y. Zhang, G.-N. Luo, G.-H. Lu, Nucl. Fusion 50 (2010) 025016.

[29] W. Xiao, W.T. Geng, Journal of Nuclear Materials 430 (2012) 132.

[30] T. Watanabe, T. Kaneko, N. Matsunami, N. Ohno, S. Kajita, and T. Kuwabara, Journal of Nuclear Materials 463, 1049 (2015).

[31] E.A. Hodille, X. Bonnin, R. Bisson, T. Angot, C.S. Becquart, J.-M. Layet, C. Grisolia, Journal of Nuclear Materials 467 (2015) 424.

[32] B. Lipschultz, J. Roth, J.W. Davis, R.P. Doerner, A.A. Haasz, A. Kalenbach, A. Kirschner, R.D. Kolasinski, A. Loarte, V. Philipps, others, MIT Report, PSFC/RR-10-4 (2010). 


\section{Figure captions:}

Figure 1: TPD measurements of deuterium retention for some of the lowest deuterium fluences. The deuterium desorption rate as a function of sample temperature is shown here, accounting for $\mathrm{D}_{2}$ and HD molecular products. Background QMS signal has not been subtracted. TPD curves for each fluences are obtained after binning of the QMS signal of individual TPD measurements in $5 \mathrm{~K}$ temperature intervals and averaging between replicate TPD measurements.

Figure 2: Deuterium retention in polycrystalline tungsten as a function of deuterium ion fluence. Blue solid square, Ogorodnikova et al. [17]. Green solid circle, this work: implantation temperature uncertainty and fluence error bars are twice the sample standard deviation from replicate TPD measurements; retention error bars represent $90 \%$ confidence interval obtained on 3 samples from the same batch. Red open diamond, this work, absolute retention from NRA, error bars are obtained from counting statistics. The grey dashed line corresponds to the SRIM implantation probability obtained for a pure tungsten sample exposed to implantation conditions of this work. The dotted black line represents the experimental power law relationship between deuterium retention $(r)$ and deuterium ion fluence $(f): r=5.65 \times 10^{5} \times f^{0.645}$.

Figure 3: Evolution of the quantity of retained deuterium in polycrystalline tungsten samples as a function of storage time (in UHV) between the end of ion implantation and the beginning of the TPD measurement. Error bars are twice the sample standard deviation from replicate measurements. The solid green line corresponds to the adjustment with Equation 1 with $B=S$ and $\tau=19$ hours. 
Figure 1

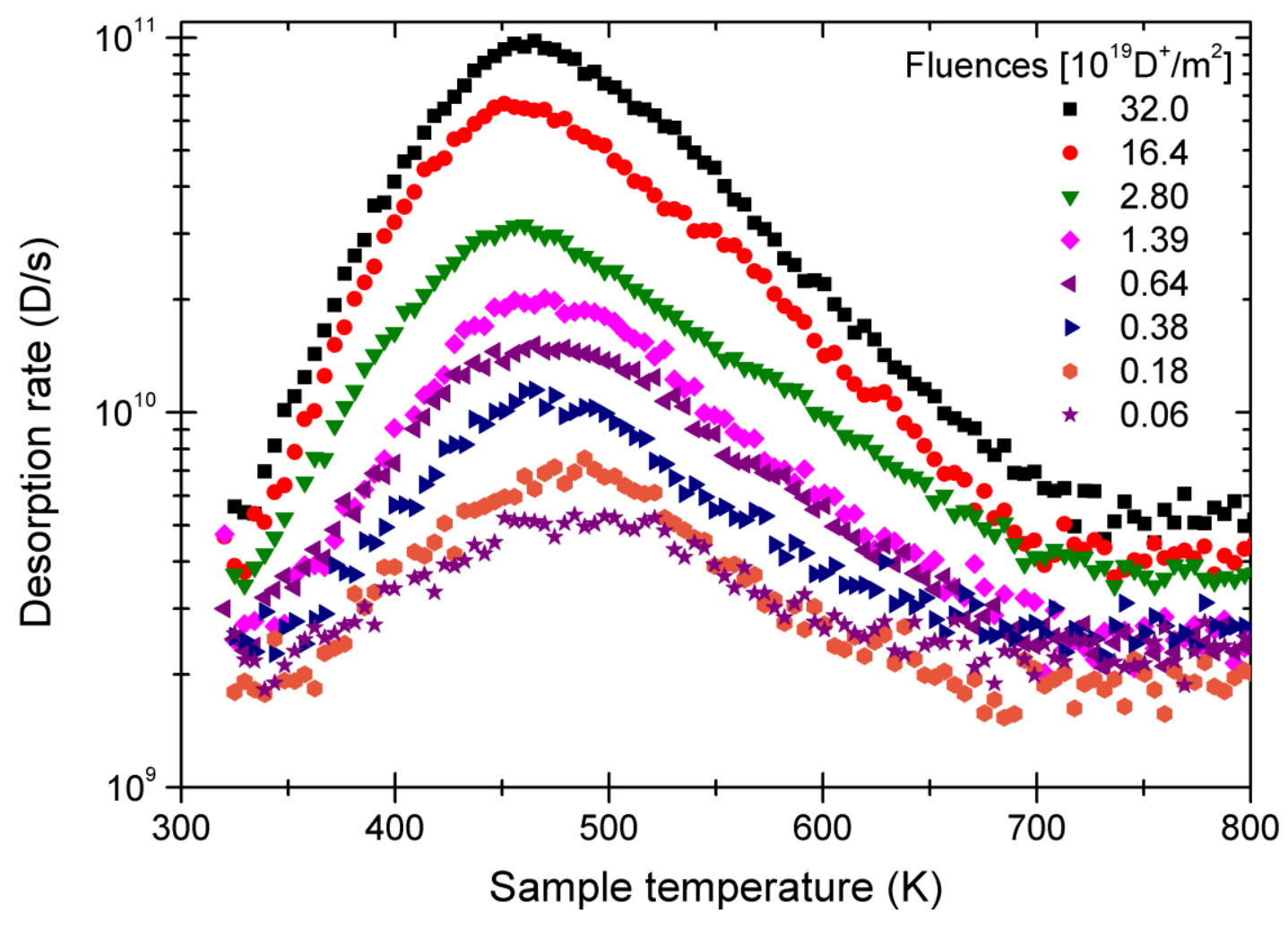


Figure 2

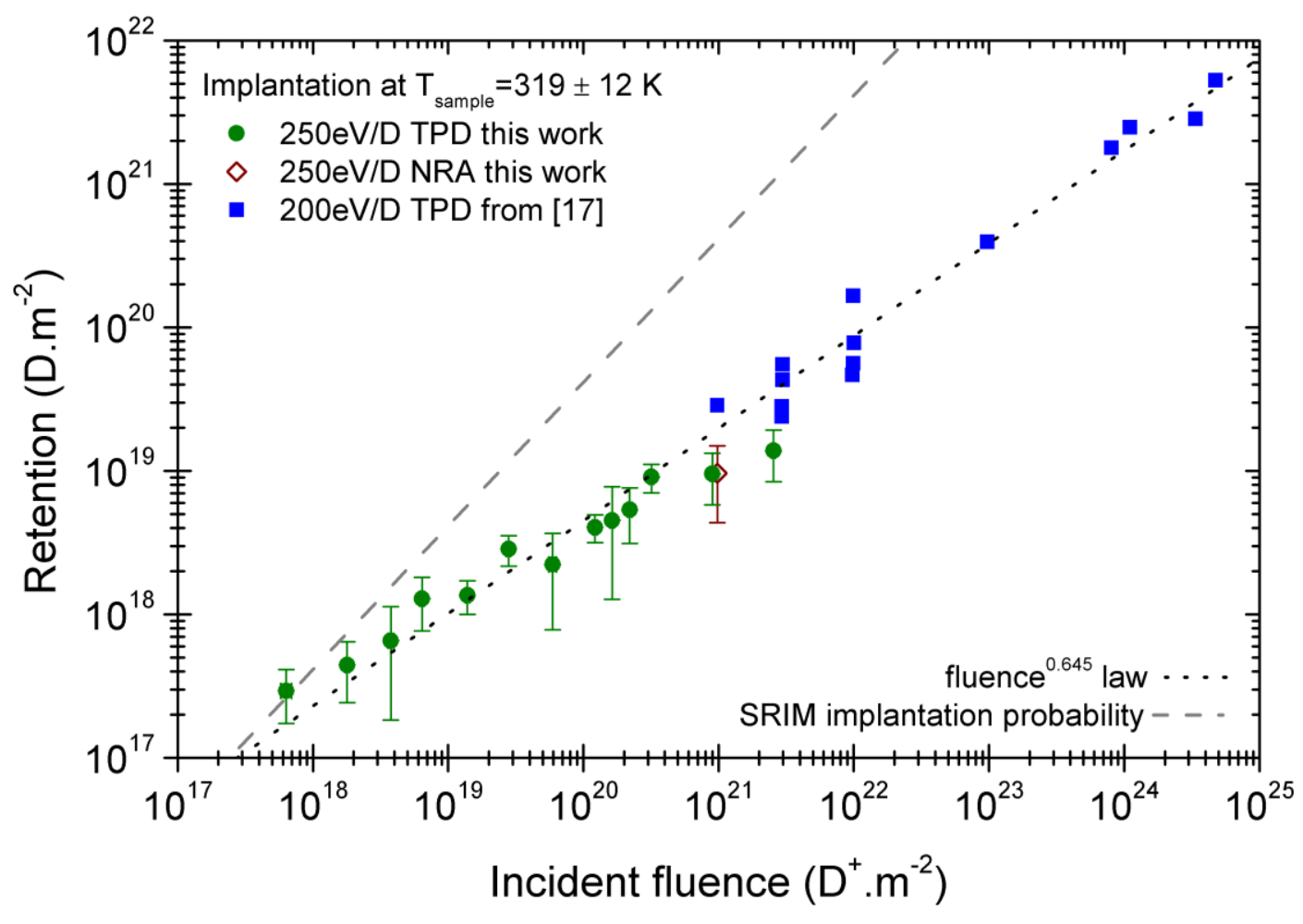


Figure 3

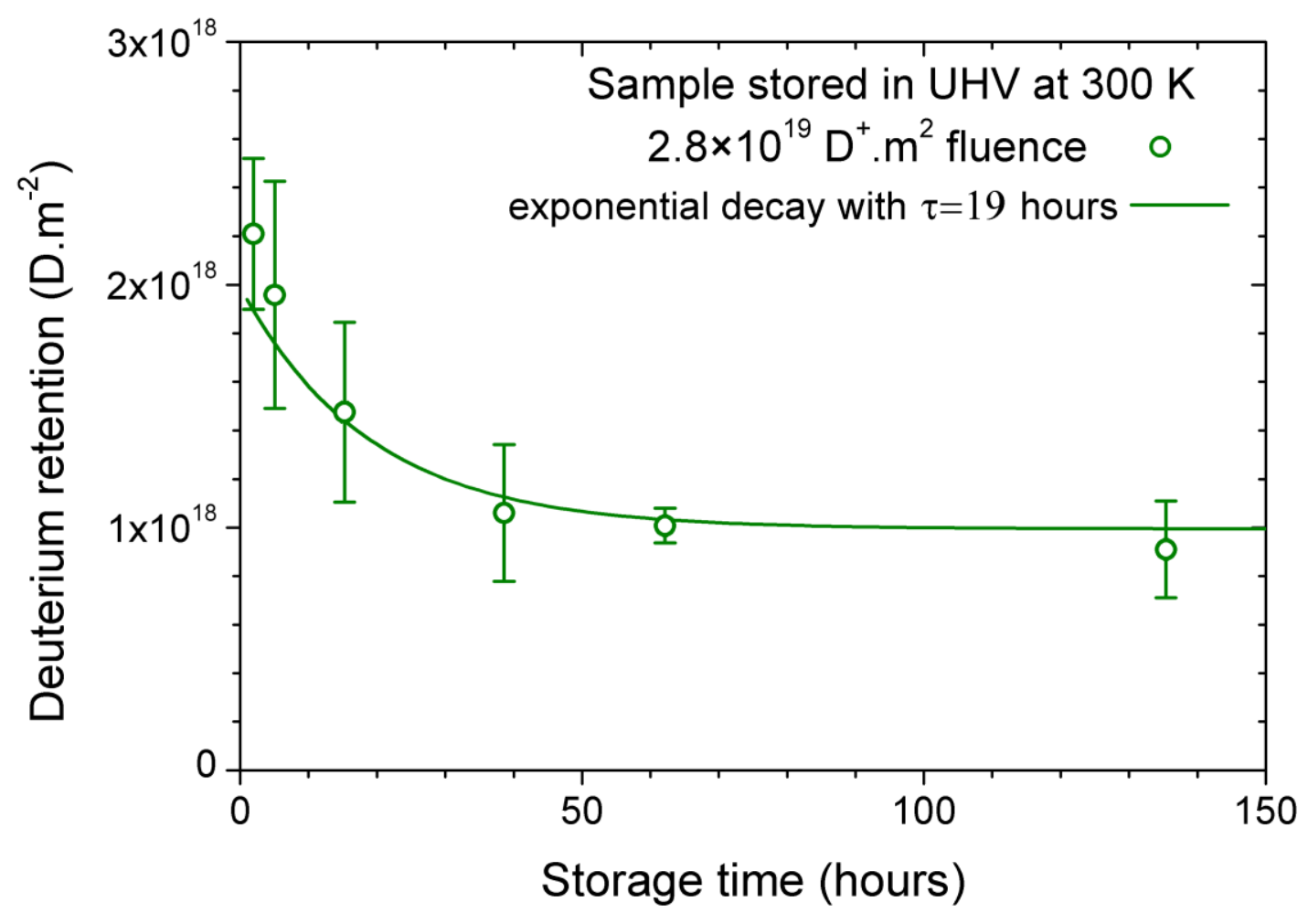

